\title{
Metal-Catalyst-Free Growth of Silica Nanowires and Carbon Nanotubes Using Ge Nanostructures
}

\author{
Takashi Uchino*, John L. Hutchison ${ }^{1}$, Greg N. Ayre, David C. Smith, \\ Kees de Groot, and Peter Ashburn
}

Faculty of Physical and Applied Sciences, University of Southampton, Southampton, SO17 1BJ, United Kingdom

${ }^{1}$ Department of Materials, University of Oxford, Parks Road, Oxford, OXI 3PH, United Kingdom

\section{Abstract}

The use of Ge nanostructures is investigated for the metal-catalyst-free growth of silica nanowires and carbon nanotubes (CNTs). Silica nanowires with diameters of $10-50 \mathrm{~nm}$ and lengths of $\leq 1 \mu \mathrm{m}$ were grown from SiGe islands, Ge dots, and $\mathrm{Ge}$ nanoparticles. High-resolution transmission electron microscopy (HRTEM) and energy dispersive X-ray spectroscopy (EDS) reveal that the nanowires grow from oxide nanoparticles on the sample surface. We propose that the growth mechanism is thermal diffusion of oxide through the $\mathrm{GeO}_{2}$ nanostructures. CNTs with diameters $0.6-2.5 \mathrm{~nm}$ and lengths of less than a few $\mu \mathrm{m}$ were similarly grown by chemical vapor deposition from different types of Ge nanostructures. Raman measurements show the presence of radial breathing mode peaks and the absence of the disorder induced D-band, indicating single walled CNTs with a low defect density. HRTEM images reveal that the CNTs also grow from oxide nanoparticles, comprising a mixture of $\mathrm{GeO}_{2}$ and $\mathrm{SiO}_{2}$.

\footnotetext{
*E-mail address: tu@ecs.soton.ac.uk
} 


\section{Introduction}

Recently, nanowires and nanotubes are attracting a lot of interest because of their potential uses in nanoelectronic and optoelectronic devices. Silica nanowires have potential applications in Si-based optoelectronics, where for example they could be used as on-chip optical interconnects or light emitters. ${ }^{1)}$ Interest in this latter application has been fuelled by the discovery of stable and strong blue light emission from silica nanowires. ${ }^{1)}$ Intensive research has been carried out on the growth of silica nanowires using techniques such as pulsed laser ablation, ${ }^{2)}$ catalytic chemical vapor deposition $(\mathrm{CVD}),{ }^{3)}$ and carbothermal reduction. ${ }^{4)}$ At the same time, the growth mechanism has been investigated and several growth models have been proposed, such as the vapor-liquid-solid (VLS) model, ${ }^{1,}$ 5) the vapor-solid (VS) model, ${ }^{6}$ and the solid-liquid-solid (SLS) model. ${ }^{7}$ However the growth mechanism is still poorly understood.

Carbon nanotubes (CNTs) have also been receiving considerable attention due to their potential for applications such as flexible thin-film transistors, ${ }^{8)}$ chemical sensors, ${ }^{9)}$ and nanomemories. ${ }^{10)}$ The interesting combination of silica nanowire waveguides, CNT devices, and complementary metal-oxide-semiconductor (CMOS) has the potential to deliver advanced CMOS integrated optoelectronics systems. The growth of nanowires and CNTs traditionally involves the use of metal nanoparticles as a catalyst. However, the integration of metal catalyzed growth with the front end of a CMOS manufacturing process is problematic due to the metal contamination. Transition metals such as Fe and $\mathrm{Ni}$ create deep levels in the Si bandgap which act as recombination centers just like Au. To reap the maximum benefits of Si very-large-scale integration (VLSI) technologies, metal-catalyst-free growth of nanowires and CNTs is required for compatibility with 
CMOS front end processing. An approach using SiC has been proposed as one solution for avoiding metal contamination, ${ }^{11,}{ }^{12}$ but this technique requires expensive $\mathrm{SiC}$ substrates and high temperature annealing over $1500^{\circ} \mathrm{C}$. Our earlier work demonstrated that silica nanowires and CNTs could be grown on SiGe substrates, ${ }^{13)}$ but the mechanism of the growth was not clear because the use of a continuous SiGe layer made it difficult to identify surface features that might give rise to the nucleation of the silica nanowires or CNTs.

In this paper, the growth mechanisms of silica nanowires and CNTs from different Ge nanostructures are investigated. A comparison is made of silica nanowires and CNTs grown from a SiGe layer, Ge dots and Ge nanoparticles produced by ion implantation and thin film deposition. The nanowires and CNTs are characterized using field-emission scanning electron microscopy (FE-SEM), high-resolution transmission electron microscopy (HRTEM), energy dispersive X-ray spectroscopy (EDS), photoluminescence (PL) spectroscopy, and Raman spectroscopy. HRTEM measurements reveal that the nanowires grow from oxide nanoparticles on the sample surface, and hence we propose that the growth is associated with the thermal diffusion of oxide through the Ge nanostructures. Raman measurements on the CNTs show that the radial breathing mode (RBM) peaks are present and the disorder induced D-band is absent, indicating single walled CNTs (SWNTs) with a low defect density. HRTEM measurements reveal that CNTs also grow from the oxide nanoparticles.

\section{Experimental Procedure}

Table I summarizes the Ge nanostructures used as the starting material for the growth of silica nanowires and CNTs. For the SiGe islands and Ge dots, low-pressure 
CVD was used to produce Ge nanostructures after the growth of a thin Si buffer layer. To accommodate the stress resulting from the lattice mismatch between $\mathrm{Si}$ and $\mathrm{Ge}$, the SiGe layer forms islands on top of the thin wetting layer and the Ge layer forms self-assembled Ge dots. The SiGe islands have heights ranging from 20 to $50 \mathrm{~nm}^{13)}$ and the Ge dots have diameters ranging from 20 to $250 \mathrm{~nm}$ and heights ranging from 10 to $25 \mathrm{~nm}^{14)}$ Then the wafers were implanted with carbon at a dose of $3 \times 10^{16} \mathrm{~cm}^{-2}$ and an energy of $30 \mathrm{keV}$. The wafers were cut into $5 \times 7 \mathrm{~mm}$ pieces and dipped in buffered hydrofluoric acid (HF) solution to remove the native oxide. Chemical oxidation was performed prior to nanowire and CNT growth using a $30 \% \mathrm{H}_{2} \mathrm{O}_{2}$ solution and samples were dried with $\mathrm{N}_{2}$ immediately without water rinse.

Ion implanted Ge nanoparticles were produced by first growing a 30-nm-thick $\mathrm{SiO}_{2}$ layer and then implanting Ge at a dose of $5 \times 10^{15} \mathrm{~cm}^{-2}$ and an energy of $20 \mathrm{keV}$. The wafers were annealed in a $\mathrm{N}_{2}$ atmosphere at $600^{\circ} \mathrm{C}$ for 40 min to create the $\mathrm{Ge}$ nanoparticles. This step was followed by a $\mathrm{HF}$ vapor etch to remove the $\mathrm{SiO}_{2}$ and expose the Ge nanocrystals formed during $\mathrm{N}_{2}$ annealing. Atomic force microscopy (AFM) measurements show that this process delivers particle heights between 1.3 and $2.9 \mathrm{~nm}$ and a mean particle density of $460 \pm 30 \mathrm{particles} / \mu \mathrm{m}^{2}{ }^{15)}$ Selected wafers were implanted with carbon at a dose of $3 \times 10^{16} \mathrm{~cm}^{-2}$ and an energy of $30 \mathrm{keV}$. For carbon implanted Ge nanoparticles, samples show a lower modal height of $0.7 \mathrm{~nm}$ and a lower particle density of $64 \pm 18$ particles $/ \mu \mathrm{m}^{2}$. We believe that the carbon ions sputter the Ge nanocrystals from the surface, thereby lowering both their density and average size.

Ge nanoparticles were also produced by depositing a 3-nm-thick Ge layer on $\mathrm{SiO}_{2}$ by either sputtering or evaporation. The nanoparticles were formed during an anneal at $850^{\circ} \mathrm{C}$ for $20 \mathrm{~min}$ in a mixture of $\mathrm{Ar}(1000 \mathrm{sccm})$ and $\mathrm{H}_{2}(300 \mathrm{sccm})$ after a pre-anneal 
in $\mathrm{H}_{2}(1000 \mathrm{sccm})$ at $950^{\circ} \mathrm{C}$. AFM measurements show that the particle heights lie between 0.6 and $2.2 \mathrm{~nm}$ and that the mean particle density is $450 \pm 20$ particles $/ \mu \mathrm{m}^{2}$.

Silica nanowires were grown from these Ge nanostructures at a temperature between 850 and $1000^{\circ} \mathrm{C}$ in $\mathrm{Ar} / \mathrm{H}_{2}(1000 / 300 \mathrm{sccm})$ for $10 \mathrm{~min}$ using a hot-wall reactor at atmospheric pressure. CNTs were grown by thermal CVD using a mixture of $\mathrm{CH}_{4} / \mathrm{H}_{2}$ $(1000 / 300 \mathrm{sccm})$ after silica nanowire growth, which was performed by annealing in either $\mathrm{Ar} / \mathrm{H}_{2}(1000 / 300 \mathrm{sccm})$ or $\mathrm{H}_{2}(1000 \mathrm{sccm})$ at a temperature between 850 and $1000^{\circ} \mathrm{C}$. A typical CNT growth condition was $850^{\circ} \mathrm{C}$ for $20 \mathrm{~min}$.

The synthesized nanowires and CNTs were observed by means of FE-SEM and HRTEM. TEM sample preparation consisted of scraping the sample surface with a surgical blade and transference onto a carbon-coated $\mathrm{Cu}$ grid. The chemical compositions of the SEM and TEM samples were analyzed by EDS. The PL measurements were carried out using a Xe lamp (250 nm) as an excitation light source. Raman spectroscopy was performed using a micro-Raman system with excitation wavelength of 532 and $633 \mathrm{~nm}$.

\section{Results}

\subsection{Silica nanowires grown from Ge dots}

Figures 1(a) and 1(b) show typical FE-SEM image of as-grown Ge dots and nanowires on Ge dots, respectively. The as-grown Ge dots are in the form of two types cones: small pyramidal dots and larger domes which are commonly seen on the samples produced by epitaxial growth. ${ }^{16,17)}$ The diameter and height of the pyramidal dots are around 25 and $10 \mathrm{~nm}$ and of domes are 200 and $20 \mathrm{~nm}$, respectively. The surface texture after the chemical oxidation is quite similar to the as-grown samples (not shown). The 
$\mathrm{Ar} / \mathrm{H}_{2}$ annealing at $1000^{\circ} \mathrm{C}$ changes the surface texture and in particular leads to the formation of curly nanowires with diameters in the range from 10 to $50 \mathrm{~nm}$ and lengths less than $1 \mu \mathrm{m}$. Figure 1(c) shows an area with a high density of nanowires. The yield of nanowires is not uniform and some areas showed a high density of nanowires, especially near randomly distributed pits. To investigate the properties of the nanowires, the samples were subjected to HF vapor etch. Figure 1(d) shows that the nanowires were eliminated by the HF treatment, indicating the nanowires are composed of oxide.

Figure 2(a) shows an EDS spectrum of a nanowire [circled and marked \#1 in Fig. 2(b)]. The spectrum shows peaks for $\mathrm{Si}$, oxygen, and carbon, but peaks for Ge and metal are not visible. This result suggests that there is little Ge in the nanowires, although we cannot discount the possibility that a small amount is present because EDS is not able to detect the presence of Ge at levels less than about $1 \%$. Although the spot size of the EDS electron beam is slightly larger than the nanowire size and consequently it is difficult to carry out quantitative analysis, the presence of oxygen is established by the presence of a peak that is clearly distinguishable from the background. To confirm this conclusion, Fig. 2(c) shows an EDS spectrum for a region of the sample without nanowires [circled and marked \#2 in Fig. 2(b)]. In this case, it can be seen that there is no evidence of an oxygen peak. The large Si signal arises from the underlying substrate and hence it is not possible to unambiguously determine whether the oxide nanowires contain Si. However, the lack of a distinct Ge peak in Fig. 2(a) suggests that the nanowires do not contain large amounts of Ge.

Figure 3 shows TEM images of the nanowires. It is clear that the nanowires have an amorphous structure with a diameter around $20 \mathrm{~nm}$. Furthermore, there is no evidence of Ge nanoparticles or metal catalyst particles either at the tip or the base of the 
nanowires. All of the nanowires were isolated and did not form bundles. These observations are consistent with the conclusion that the nanowires are formed from an oxide. The mechanism of the growth is discussed later.

Figure 4 shows the PL spectrum of the nanowires from a region of a sample containing high density nanowires, similar to that shown in Fig. 1(c). Strong blue-green light emission at $490 \mathrm{~nm}$ is seen. Repeated measurements were made in different regions of the sample and the $490 \mathrm{~nm}$ peak was consistently seen in areas containing high density of nanowires, but not seen in areas where no nanowires were present. Lin et al. $^{4)}$ reported that $\mathrm{SiO}_{2}$ nanowires had a similar blue-green light emission at $500 \mathrm{~nm}$ due to the neutral oxygen vacancy in silica. He et al. ${ }^{18)}$ reported that SiGe oxide nanowires exhibited blue light emission with a peak at $415 \mathrm{~nm}$ whereas their silica nanowires exhibited a peak at $470 \mathrm{~nm}$. These results suggest that our nanowires are silica nanowires rather than $\mathrm{SiGe}$ or Ge oxide nanowires.

Figure 5 shows a Raman spectrum from a region of a sample containing high density nanowires, similar to that shown in Fig. 1(c). The spectrum shows the presence of a sharp peak at $485 \mathrm{~cm}^{-1}$ in addition to the Si peak at $520 \mathrm{~cm}^{-1}$. The Raman measurements were made several times on the same sample and these two peaks were consistently seen in regions of high nanowire density, but not seen in regions where no nanowires were present. Similar Raman peaks around $460 \mathrm{~cm}^{-1}$ have been reported in the literature for silica nanowires. ${ }^{1)}$ The Raman peak for silica nanowires tends to occur at a smaller wave number than that of bulk non-crystalline $\mathrm{SiO}_{2}$ which is around 500 $\mathrm{cm}^{-1} .^{19)}$ These results, together with the EDS results in Fig. 2 and the PL results in Fig. 4, lead us to conclude that our nanowires are comprised primarily of silica. 


\subsection{Silica nanowires grown from Ge nanoparticles}

To further investigate the role of Ge and oxygen in the growth of silica nanowires, we have directly fabricated Ge nanoparticles in two ways. The first method uses Ge implantation into a $\mathrm{SiO}_{2}$ layer and subsequent annealing at $600^{\circ} \mathrm{C}$ to form $\mathrm{Ge}$ nanoparticles. The Ge nanoparticles are then removed from the $\mathrm{SiO}_{2}$ layer using an $\mathrm{HF}$ vapor etch. The second method uses a thin Ge film deposited on a $\mathrm{SiO}_{2}$ layer and subsequent annealed at $950^{\circ} \mathrm{C}$ to produce the Ge nanoparticles. Figure 6 shows FE-SEM images of silica nanowires grown from Ge nanoparticles produced by Ge implantation (a) and Ge thin film deposition (b). Both types of Ge nanoparticle have produced nanowires, though the diameters and lengths of the nanowires are different for the two methods. For the Ge nanoparticles produced by ion implantation, the nanowire diameter is typically $20 \mathrm{~nm}$ and the length is typically $1 \mu \mathrm{m}$, whereas for the Ge nanoparticles produced by thin film Ge deposition and anneal, the nanowire diameter is typically 30 $\mathrm{nm}$ and the length is typically $0.2 \mu \mathrm{m}$. The nanowires grown by the former method are therefore generally longer and slightly thinner than these grown by the latter method. Experiments showed that the nanowires were eliminated by an etching in HF vapor and hence it can again be concluded that the nanowires are composed of oxide. As the Ge nanoparticle sizes are similar for both methods (Table I), this difference in nanowire geometry is probably associated with the different substrate type $\left(\mathrm{Si}\right.$ and $\left.\mathrm{SiO}_{2}\right)$. For the samples prepared using Ge implantation, the oxygen feedstock could come from both the native oxide on the Ge nanoparticles and/or the native oxide on the Si substrate. The Si feedstock could come from the Si substrate. For the samples prepared by Ge thin film deposition, the oxygen feedstock could come from both the $\mathrm{SiO}_{2}$ substrate and/or the native oxide on the Ge nanoparticles. The $\mathrm{Si}$ feedstock could come from the $\mathrm{SiO}_{2}$ 
substrate. The results in Fig. 6 suggest that a $\mathrm{Si}$ substrate is a more effective than a $\mathrm{SiO}_{2}$ substrate to grow silica nanowires.

\subsection{Carbon nanotubes grown from Ge nanostructures}

Figure 7 shows FE-SEM images of CNTs grown from different Ge nanostructures. Fig. 7(a) shows results for growth on SiGe islands. Both silica nanowires and CNTs can be seen, with many of the silica nanowires growing from the gaps between the SiGe islands. The CNTs are generally seen as straight and thin features with a diameter of less than $10 \mathrm{~nm}$ and a length of less than $5 \mu \mathrm{m}$. Figure 7(b) shows an FE-SEM image of CNTs grown from the Ge dots. Both silica nanowires and CNTs are again present, and the CNTs are seen as straight and thin features with a small diameter and the nanowires as curly and thick features with a diameter of typically $20 \mathrm{~nm}$. No CNTs were seen on samples without carbon implantation and chemical oxidation, demonstrating that carbon and oxide are necessary for CNT growth on SiGe islands and Ge dots, as reported previously. ${ }^{13,14)}$ Figures 7(c) and 7(d) show FE-SEM images after CNT growth from Ge nanoparticles produced by Ge implantation and thin Ge film deposition, respectively. Both processes deliver a higher $\mathrm{CNT}$ area density of around $3.0 \mathrm{CNTs} / \mu \mathrm{m}^{2}$ compared with the growth on SiGe islands and Ge dots which a deliver CNT area density of less than 1.0 CNTs $/ \mu \mathrm{m}^{2}$. In contrast to the results for SiGe islands and $\mathrm{Ge}$ dots, carbon implantation and chemical oxidation are not essential for CNT growth from Ge nanoparticles. $^{15)}$

Figure 8 shows Raman spectra corresponding to the sample in Fig. 7(d). The Raman spectrum in Fig. 8(a) clearly shows the RBM peaks associated with SWNTs. RBM peaks have been observed with different Raman shifts from $190 \mathrm{~cm}^{-1}$ to the lower limit 
of $120 \mathrm{~cm}^{-1}$, which is imposed by our Raman notch filter. Assuming a standard formula for converting RBM peaks to nanotube diameter, ${ }^{20)}$ the SWNTs have diameters in the range 1.3-2.0 $\mathrm{nm}$. Despite considerable effort we have been unable to detect the disorder induced D-band feature around $1350 \mathrm{~cm}^{-1}$ as shown in Fig. 8(b). This indicates that the CNTs have a low defect density. Raman spectra on CNTs grown from SiGe islands and Ge dots also showed that the disorder induced D-band was absent. ${ }^{13,14)}$

To investigate the mechanism of CNT growth from the Ge nanoparticles, the ends of CNTs were observed by HRTEM and a selection of typical results is shown in Fig. 9. We have occasionally seen CNTs grown from the nanoparticles with a diameter around $20 \mathrm{~nm}$ as shown in Fig. 9(a). This is surprising given that we would expect Ge nanoparticles with diameters less than $5 \mathrm{~nm}$ to be necessary for the growth of SWNTs. ${ }^{15)}$ Figure 9(b) shows a HRTEM image of the early stage of CNT growth [indicated by arrow in Fig. 9(b)], indicating that CNTs start to grow from an amorphous structure on the surface of the sample. Figure 9(c) shows a HRTEM image of a CNT growing from a large nanoparticle. This result is typical of many HRTEM images taken on these samples, all of which indicate that the nanoparticle is amorphous. Figure 9(d) shows an X-ray diffraction pattern from the nanoparticle, which confirms that the nanoparticle is amorphous. To investigate the composition of the amorphous nanoparticles, Fig. 10 shows EDS results for the region containing the amorphous nanoparticles. A CNT is grown from the large nanoparticle again. Peaks can be seen for $\mathrm{Si}, \mathrm{Ge}$ and oxygen, indicating that the nanoparticles are likely to be an oxide, probably comprising a mixture of $\mathrm{GeO}_{2}$ and $\mathrm{SiO}_{2}$. The other peaks for carbon and $\mathrm{Cu}$ come from the carbon coated $\mathrm{Cu}$ grid. 


\section{Discussion}

Our experiments on the growth of silica nanowires from different Ge nanostructures have shown no evidence of metal catalyst particles at either ends of the silica nanowires (Fig. 3). We can therefore conclude that the growth mechanism of the silica nanowires does not follow a regular VLS model ${ }^{1)}$ arising, for example, from unintentional metal contamination. However, for all four types of Ge nanostructure, a high temperature anneal over $850^{\circ} \mathrm{C}$ is required to grow the nanowires.

The results in Fig. 10 showed that the nanoparticles were an oxide comprised of a mixture of $\mathrm{GeO}_{2}$ and $\mathrm{SiO}_{2}$. One possible mechanism by which silica nanowires could grow from an oxide layer is that reported by LeGoues et al. ${ }^{21)}$ who showed that Ge has a catalytic effect on the oxidation of $\mathrm{Si}$ at high temperatures over $800^{\circ} \mathrm{C}$. When a $\mathrm{Si}$ substrate and $\mathrm{GeO}_{2}$ nanoparticles are close to each other, Ge atoms are replaced by $\mathrm{Si}$ atoms to form $\mathrm{SiO}_{2}$. A continuous supply of both $\mathrm{Si}$ and oxygen sources would then provide the feedstock needed for the silica nanowire growth. ${ }^{2)}$ The PL measurement result in Fig. 4 indicates that our nanowires are silica nanowires rather than SiGe oxide nanowires. This result is consistent with a mechanism of the oxidation induced Ge condensation method which is commonly used to form a relaxed SiGe layer for high-performance CMOS. ${ }^{22)}$ An alternative mechanism for the silica nanowire growth could be the carbothermal reduction ${ }^{23,24)}$ of $\mathrm{GeO}_{2}$. This mechanism is consistent with the result that a carbon implantation is needed for silica nanowire growth from SiGe islands and Ge dots. However it is not consistent with the results for silica nanowire growth from Ge nanoparticles, where a carbon implantation was not necessary. While we cannot completely eliminate the possibility of unintentional carbon contamination in the latter samples, there is no direct evidence from our results to support this 
mechanism.

The HRTEM results in Figs. 9 and 10 provide clear evidence that CNTs can grow from an oxide layer on the sample surface. The low melting point of $\mathrm{GeO}_{2}\left(400^{\circ} \mathrm{C}\right)$ nanoparticles could facilitate CNT growth. The melting of the oxide nanostructures during the pre-annealing would allow a wetting layer to form that allowed the absorption of decomposed feedstock. Precipitation of a carbon cap around the nanostructure and the subsequent formation of an early stage CNT would then explain the structure indicated by the arrow in Fig. 9(b). From these results, we speculate that once the oxide nanostructures have melted during the pre-annealing and formed a wetting layer, decomposed feedstock is absorbed into the wetting layer and precipitated as a carbon cap around the nanostructure, which then grows to form CNTs. In our earlier work on CNT growth using Ge nanoparticles, a higher CNT density was obtained after silica nanowire growth, ${ }^{15)}$ indicating that the growth of CNTs may be influenced by the presence of oxide nanowires. This trend is in good agreement with the recent experimental results of Liu et al. ${ }^{25)}$ and Huang et al. ${ }^{26)}$ who reported on CNT growth from oxide nanoparticles. They separately demonstrated CNT growth from $\mathrm{SiO}_{2}$ nanoparticles formed by scratching the surface of a $\mathrm{SiO}_{2}$ layer. Whilst the growth of CNTs is possible using this method, it is not a practical growth method for integrating CNTs into CMOS technology. Takagi et al. ${ }^{27)}$ reported that the yield of CNTs grown from $\mathrm{Ge}$ nanoparticles was higher than $\mathrm{Si}$ and $\mathrm{SiC}$ nanoparticles, suggesting that materials with lower melting points are more likely seeds for CNT growth. From this point of view, $\mathrm{GeO}_{2}$ is more suitable for CNT growth than $\mathrm{SiO}_{2}$ (melting point $1650^{\circ} \mathrm{C}$ ).

Although further research is needed to integrate our growth method into a CMOS fabrication process, our results nevertheless demonstrate a practical method of growing 
CNTs without a metal catalyst.

\section{Conclusions}

We have developed metal-catalyst-free growth methods for silica nanowires and CNTs using Ge nanostructures. HRTEM measurements have shown that the silica nanowires have diameters ranging from 10 to $50 \mathrm{~nm}$ and lengths less than $1 \mu \mathrm{m}$ and that the nanowires grow from oxide nanostructures on the sample surface. We propose that the growth is associated with the thermal diffusion of oxide through the $\mathrm{GeO}_{2}$ nanostructures. CNTs with diameters in the range from 0.6 to $2.5 \mathrm{~nm}$ and lengths less than a few $\mu \mathrm{m}$ were also synthesized using CVD from Ge nanostructures. Raman measurements showed that the RBM peaks were present and the disorder induced D-band was absent, indicating SWNTs with a low defect density. HRTEM and EDS measurements revealed that the CNTs grow from oxide nanoparticles, comprised of a mixture of $\mathrm{GeO}_{2}$ and $\mathrm{SiO}_{2}$.

\section{Acknowledgement}

The authors acknowledge EPSRC for supporting this work. 


\section{References}

1) D. Yu, Q. Hang, Y. Ding, H. Zhang, Z. Bai, J. Wang, Y. Zou, W. Qian, G. Xiong, and S. Feng: Appl. Phys. Lett. 73 (1998) 3076.

2) I. Aharonovich, Y. Lifshitz, and S. Tamir: Appl. Phys. Lett. 90 (2007) 263109.

3) S. Sun, G. Meng, M. Zhang, Y. Tian, T. Xie, and L. Zhang: Solid State Commun. 128 (2003) 287.

4) Y. Lin and W. Lin: Nanotechnology 16 (2005) 1648.

5) D. Sood, P. Sehhar, and S. Bhansali: Appl. Phys. Lett. 88 (2006) 143110.

6) Y. Zhang, N. Wang, R. He, J. Liu, X. Zhang, and J. Zhu: J. Cryst. Growth 233 (2001) 803.

7) C. Wang, L. Chan, D. Xiao, T. Lin, and H. Shih: J. Vac. Sci. Technol. B 24 (2006) 613.

8) Q. Cao, H. Kim, N. Pimparkar, J. Kulkarni, C. Wang, M. Shim, K. Roy, M. Alam, and J. Rogers: Nature 454 (2008) 495.

9) J. Kong, N. Franklin, C. Zhou, S. Peng, J. Cho, and H. Dai: Science 287 (2000) 622.

10) T. Rueckes, K. Kim, E. Joselevich, G. Tseng, C. Cheung, and C. M. Lieber: Science 289 (2000) 94.

11) M. Kusunoki, M. Rokkaku, and T. Suzuki: Appl. Phys. Lett. 71 (1997) 2620.

12) H. Takikawa, M. Yatsuki, and T. Sakakibara: Jpn. J. Appl. Phys. 38 (1999) L401.

13) T. Uchino, K. N. Bourdakos, C. H. Groot, P. Ashburn, M. E. Kiziroglou, D. G. Dilliway, and D. C. Smith: Appl. Phys. Lett. 86 (2005) 233110.

14) T. Uchino, K. N. Bourdakos, C. H. Groot, P. Ashburn, S. Wang, M. E. Kiziroglou, D. G. Dilliway, and D. C. Smith: Proc. IEEE Conf. Nanotechnology, 2005, 
WE-PS3-4.

15) T. Uchino, G. Ayre, D. C. Smith, J. L. Hutchison, C. H. Groot, and P. Ashburn: J. Electrochem. Soc. 156 (2009) K144.

16) L. Vescan, T. Stoica, O. Cheretien, M. Goryll, E. Mateeva, and A. Muck: J. Appl. Phys. 87 (2000) 7275.

17) G. Ribeiro, A. Bratkovski, T. Kamins, D. Ohlberg, and R. Williams: Science 279 (1998) 353.

18) J. He, W. Wu, S. Lee, L. Chen, Y. Chueh, and L. Chou: Appl. Phys. Lett. 86 (2005) 263109.

19) T. Nakano, N. Mura, and A. Tsuzumitani: Jpn. J. Appl. Phys. 34 (1995) L1064.

20) A. Jorio, C. Fantini, M. Dantas, M. Pimenta, A. Filho, G. Samsonidze, V. Brar, G. Dresselhaus, M. Dresselhaus, A. Swan, M. Unlu, B. Goldberg, and R. Saito: Phys. Rev. B 66 (2002) 115411.

21) F. LeGoues, R. Rosenberg, and B. Meyerson: Appl. Phys. Lett. 54 (1989) 644.

22) T. Tezuka, N. Sugiyama, T. Mizuno, M. Suzuki, and S. Takagi: Jpn. J. Appl. Phys. 40 (2001) 2866.

23) P. Carter, B. Gleeson, and D. Young: Oxid. Met. 56 (2001) 375.

24) S. Li, X. Zhu, and Y. Zhao: J. Phys. Chem. B 108 (2004) 17032.

25) B. Liu, W. Ren, L. Gao, S. Li, S. Pei, C. Liu, C. Jiang, and H. Cheng: J. Am. Chem. Soc. 131 (2009) 2082.

26) S. Huang, Q. Cai, J. Chen, Y. Qian, and L. Zhang: J. Am. Chem. Soc. 131 (2009) 2094.

27) D. Takagi, H. Hibino, S. Suzuki, Y. Kobayashi, and Y. Homma: Nano Lett. 7 (2007) 2272. 


\section{Figure Captions}

Fig. 1. (Color online) FE-SEM images of (a) as-grown Ge dots on a Si substrate, (b) nanowires on and around Ge dots, (c) an area of high nanowire density and (d) the sample after HF vapor etch. The circles in (c) and (d) show pits.

Fig. 2. (Color online) SEM/EDS analysis of nanowires grown from $\mathrm{Ge}$ dots on a $\mathrm{Si}$ substrate. (a) EDS spectra of a nanowire. (b) SEM image of the nanowires where the EDS measurements were carried out. (c) EDS spectra of the Si substrate.

Fig. 3. HRTEM images of nanowires grown from Ge dots on a Si substrate; (a) initial stage of nanowire growth and (b) the tip of the nanowire.

Fig. 4. Photoluminescence spectrum of nanowires grown from $\mathrm{Ge}$ dots on a $\mathrm{Si}$ substrate measured with a Xe lamp $(250 \mathrm{~nm})$ as an excitation light source.

Fig. 5. Raman spectra of nanowires grown from Ge dots on a Si substrate measured with $532 \mathrm{~nm}$ excitation.

Fig. 6. FE-SEM images of silica nanowires grown from Ge nanoparticles produced by (a) Ge implantation into a $\mathrm{SiO}_{2}$ layer and anneal and removal of the $\mathrm{SiO}_{2}$ layer using an $\mathrm{HF}$ vapor etch (b) Ge thin film deposition on $\mathrm{SiO}_{2}$ substrate and anneal.

Fig. 7. FE-SEM images of CNTs grown from different Ge nanostructures; (a) a SiGe layer; (b) Ge dots; (c) Ge nanoparticles produced by Ge implantation; (d) Ge thin film deposition.

Fig. 8. Raman spectra of the CNTs shown in Fig. 7(d) measured with $633 \mathrm{~nm}$ excitation. These CNTs were grown from Ge nanoparticles produced by thin film Ge deposition and anneal. (a) An RBM feature and (b) a G-band feature. The lack of a D-band peak around $1350 \mathrm{~cm}^{-1}$ and strong RBM peaks indicate that high quality SWNTs are present. 
Fig. 9. (Color online) (a) FE-SEM image of a CNT and Ge nanoparticles. (b) HRTEM image of SWNTs grown from Ge nanoparticles. The arrow shows a CNT in the early stages of growth. (c) HRTEM image of CNTs growing from an amorphous nanoparticle. (d) The corresponding Fourier transform image of the nanoparticle.

Fig. 10. (Color online) EDS spectrum of a nanoparticle from which a CNT has grown. The inset shows a HRTEM image of a CNT and the nanoparticle where the EDS measurements were carried out. 
Table I. Summary of Ge nanostructures used for silica nanowire and CNT growth.

\begin{tabular}{lcl}
\hline \multicolumn{1}{c}{ Ge nanostructures } & Substrate & \multicolumn{1}{c}{ Pre-treatment } \\
\hline SiGe islands: $20-50 \mathrm{~nm}$ height, $\mathrm{Ge}=30 \%$ & $\mathrm{Si}$ & $\begin{array}{l}\text { Carbon implantation }+ \\
\text { chemical oxidation }\end{array}$ \\
Ge dots: $10-25 \mathrm{~nm}$ height, $20-250 \mathrm{~nm}$ in diameter & $\mathrm{Si}$ & $\begin{array}{l}\text { Carbon implantation }+ \\
\text { chemical oxidation }\end{array}$ \\
$\begin{array}{l}\text { Ge nanoparticles: } 1.3-2.9 \mathrm{~nm} \text { height }(\mathrm{Ge} \text { implantation } \\
\left.\text { into } \mathrm{SiO}_{2}+\mathrm{N}_{2} \text { annealing at } 600^{\circ} \mathrm{C}\right)\end{array}$ & $\mathrm{Si}$ & HF vapor etch \\
$\begin{array}{l}\text { Ge nanoparticles: } 0.6-2.2 \mathrm{~nm} \text { height }(3 \mathrm{~nm} \text {-thick } \mathrm{Ge} \\
\left.\text { film deposition }+\mathrm{Ar} \text { annealing at } 950^{\circ} \mathrm{C}\right)\end{array}$ & $\mathrm{SiO}_{2}$ & None \\
\hline \hline
\end{tabular}




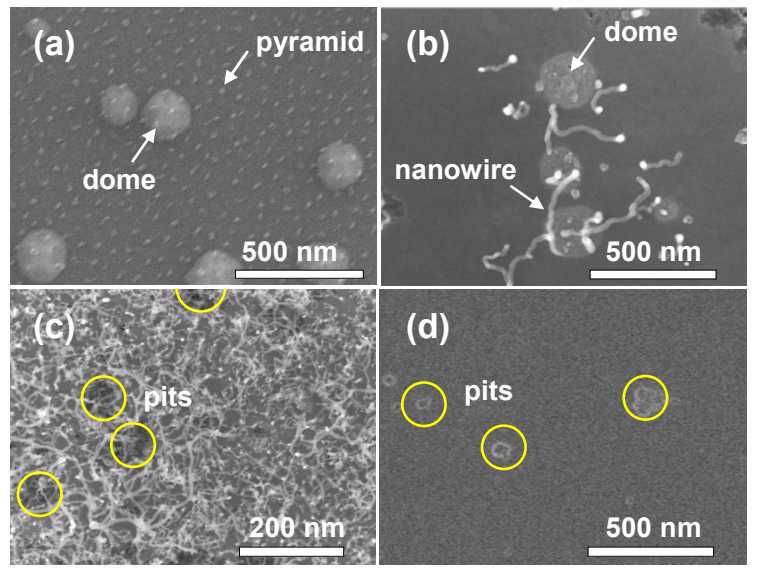

Fig. 1. T. Uchino et al. 


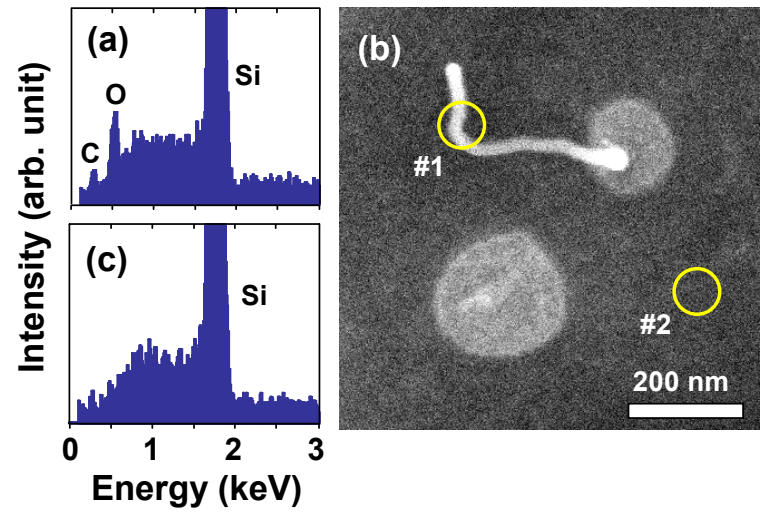

Fig. 2. T. Uchino et al. 

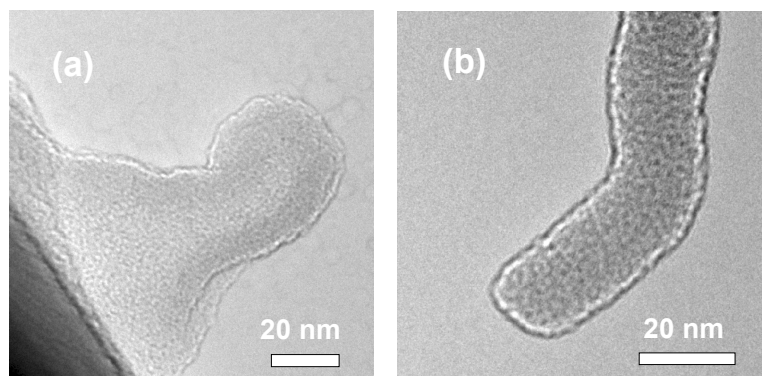

Fig. 3. T. Uchino et al. 


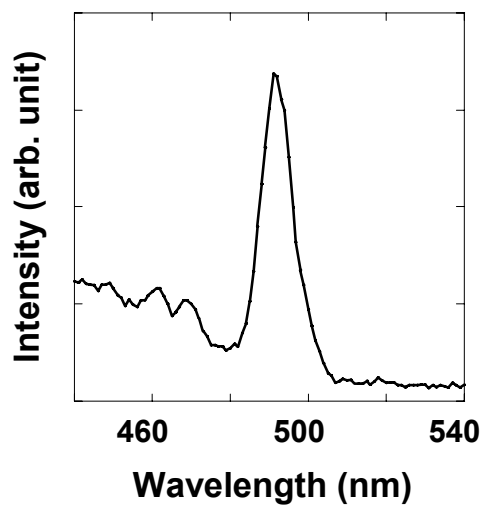

Fig. 4. T. Uchino et al. 


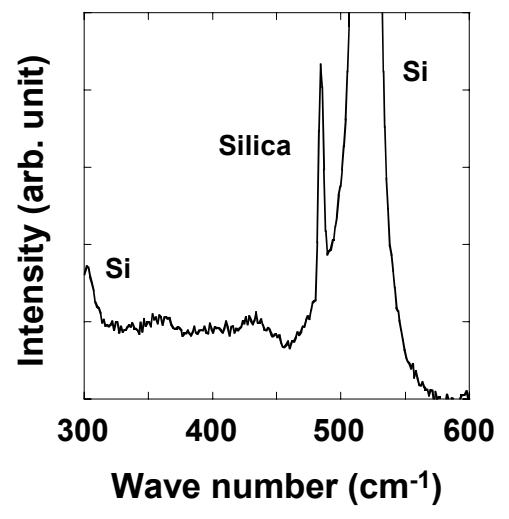

Fig. 5. T. Uchino et al. 


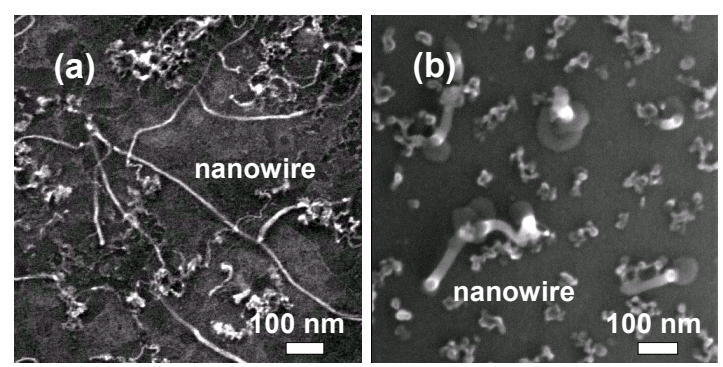

Fig. 6. T. Uchino et al. 


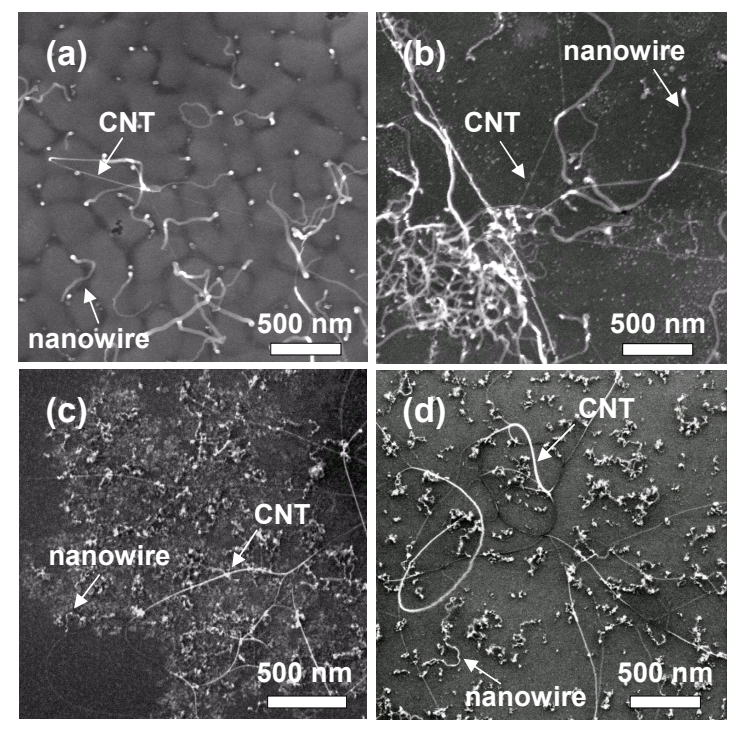

Fig. 7. T. Uchino et al. 


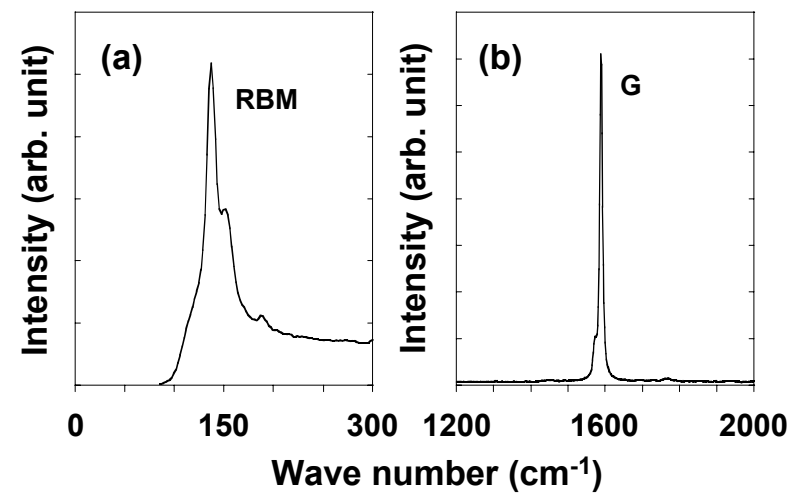

Fig. 8. T. Uchino et al. 

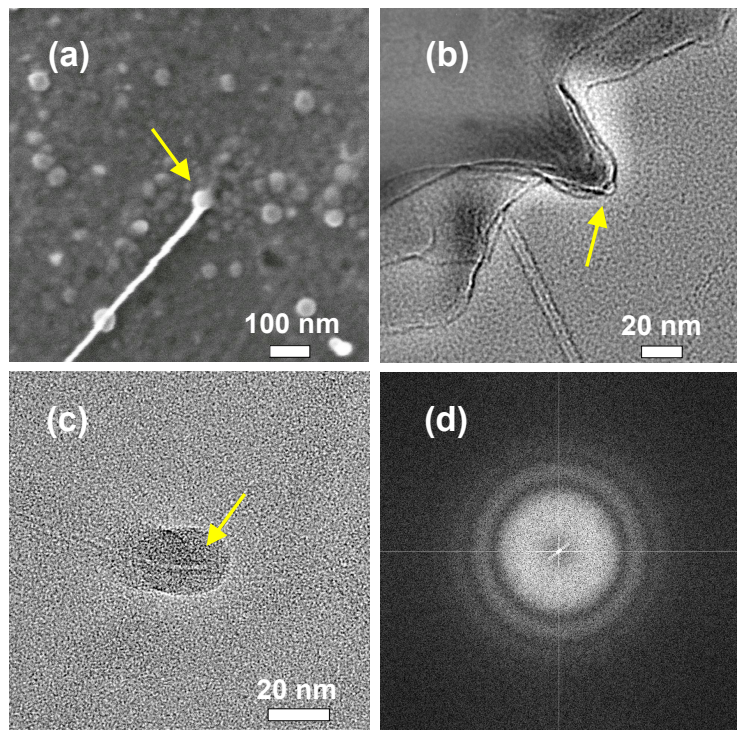

Fig. 9. T. Uchino et al. 


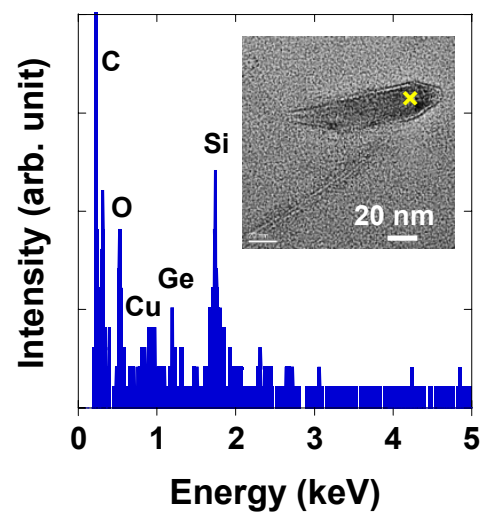

Fig. 10. T. Uchino et al. 